\title{
Tricuspid valve regurgitation in the presence of endocardial leads - an underestimated problem
}

\author{
Anna Rydlewska ${ }^{1,2}$, Andrzej Ząbek ${ }^{1}$, Krzysztof Boczar ${ }^{1}$, Jacek Lelakowski ${ }^{1,2}$, Barbara Małecka ${ }^{1,2}$ \\ ${ }^{1}$ Department of Electrocardiology, John Paul II Hospital, Krakow, Poland \\ ${ }^{2}$ Institute of Cardiology, Jagiellonian University Medical College, Krakow, Poland
}

Adv Interv Cardiol 2017; 13, 2 (48): 165-169

DOI: https://doi.org/10.5114/pwki.2017.68073

\section{Introduction}

In about $80 \%$ of the population, a small degree of tricuspid insufficiency occurs and therefore is considered almost physiological [1]. Higher mortality has been described among patients with moderate and severe tricuspid regurgitation compared to patients without it, irrespective of the pulmonary pressure value or systolic function of the left and right ventricle [2-4]. A fourfold increase in the risk of substantial tricuspid regurgitation is caused by the presence of the endocardial lead [5].

A chest X-ray examination is used in order to assess the position of the lead in the heart [6]. The influence of endocardial lead position in the chest X-ray on tricuspid valve function and the presence of its insufficiency has not yet been described in the literature.

\section{Aim}

The aim of the study was to determine the correlation between the radiological endocardial lead position and the tricuspid valve regurgitation severity.

\section{Material and methods}

The study comprised 100 consecutive, random patients who were admitted to the outpatient clinic between November 2013 and September 2014 for a routine follow-up after cardiac stimulation system implantation. There were no data available about their pre-implantation echocardiographic examination or the lead position directly after implantation.

Inclusion criteria: patient after cardiac stimulation system implantation; and, conscious consent for the trial.

Exclusion criteria: severe mitral valve insufficiency and mitral valve prosthesis.
The patient's chest X-ray was taken in the postero-anterior (PA) and lateral position, and transthoracic echocardiography was performed.

The authors introduced the definitions of optimal and non-optimal lead position.

An optimal lead position in the PA radiological picture was defined as its free transition through the right heart chambers and through the tricuspid valve as an arch with its curve facing the diaphragm.

An excessive lead length was defined as its double-crossing through the valve or creating a loop at the valve level (Figure $1 \mathrm{~A}$ ), transition of two parallel leads through the valve creating an expansion mechanism (Figure $1 \mathrm{~B}$ ), or excessive lead length in the right atrium with the lead loop surrounding the inferior wall of the atrium and creating an arch facing upright at the tricuspid valve level (Figure $1 \mathrm{C}$ ).

Insufficient lead length was defined as excessive strengthening of the lead at the tricuspid valve level, with stiff, linear passage between the lead tip and the right atrium entrance (Figure $1 \mathrm{D}$ ).

Radiological assessment of the lead position was performed by two doctors performing implantation procedures for over 5 years - not optimal lead position was recognized at their consensual opinion. Echocardiographic examination evaluated:

- tricuspid valve function, with severe regurgitation defined according to the ESC guidelines [7];

- mitral valve function;

- heart chambers' diameters: left ventricle end diastolic diameter (LVEDd); end diastolic right ventricle diameter (RVEDd) (in parasternal longitudinal view - 1, in a four-chamber apical view: at heart base - 2; at chordae tendineae attachment - 3, in longitudinal axis -4); size of both atria (left atrium - LA; right atrium - RA);

\section{Corresponding author:}

Andrzej Ząbek MD, PhD, MSc, Department of Electrocardiology, John Paul II Hospital, 80 Prądnicka St, 31-202 Krakow, Poland, phone: +48 694194 757, e-mail: andrzej___z@poczta.onet.pl

Received: 14.09.2016, accepted: 30.12.2016. 

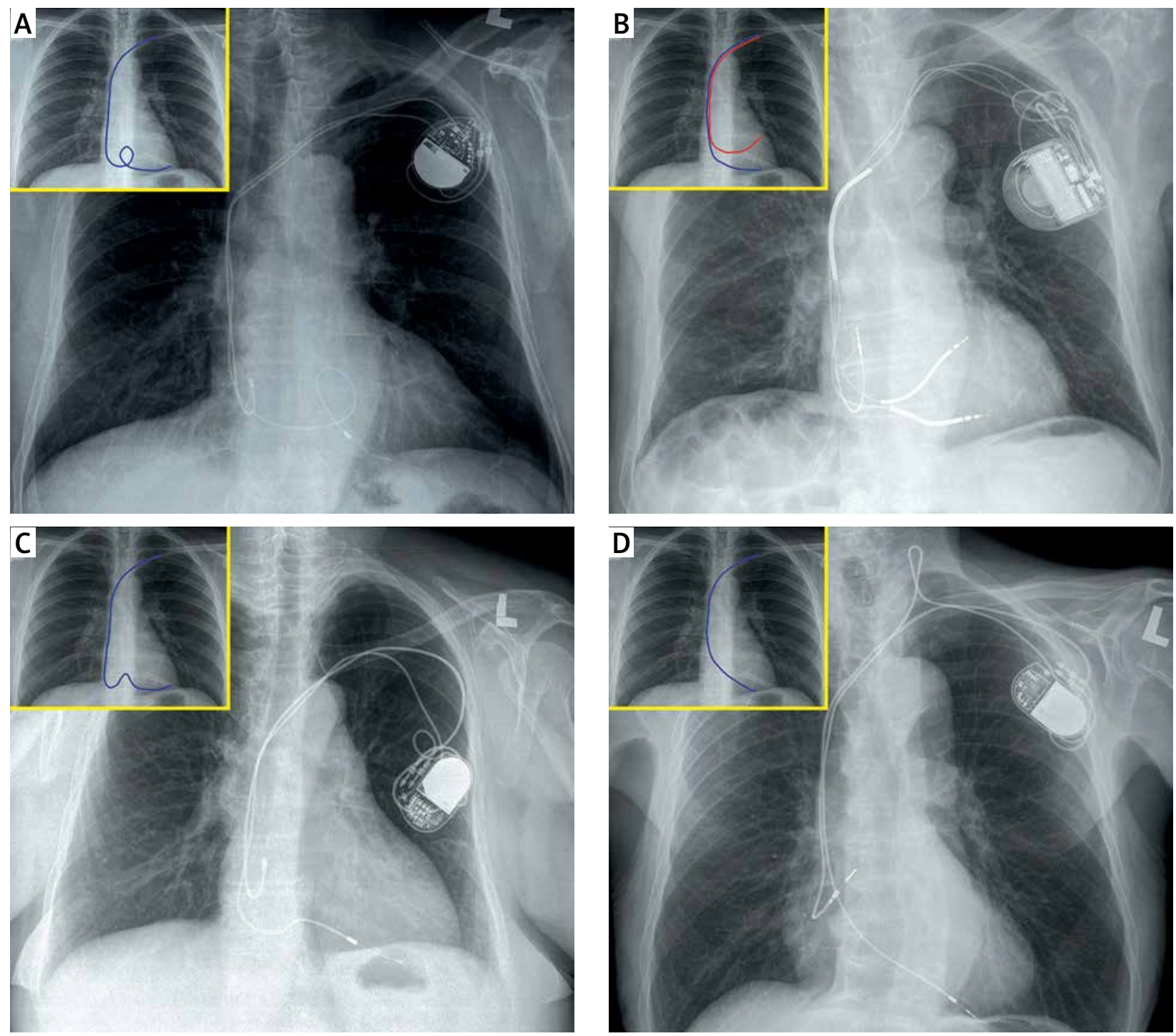

Figure 1. Chest X-ray: A-C: An excessive lead length (A - its double-crossing through the valve or creating a loop at the valve level, B - transition of two parallel leads through the valve creating the expansion mechanism, $\mathbf{C}$ - excessive lead length in the right atrium with the lead loop surrounding the inferior wall of the atrium and creating an arch facing upright at the tricuspid valve level). D - Insufficient lead length: excessive strengthening of the lead at the tricuspid valve level, with stiff, linear passage between the lead tip and the right atrium entrance

- left ventricle systolic function (LVEF) (left ventricle ejection fraction with Simpson method); and

- left ventricle diastolic function (E/A assessment).

The end point of the study was confirmation of severe tricuspid regurgitation.

Patients were divided into two groups: group A presence of severe tricuspid regurgitation; group $B-a b$ sence of severe tricuspid regurgitation.

The two groups were compared according to the demographic, clinical, echocardiographic, and stimulation system technical parameters.

Analyzed parameters were: demographic: age, gender; clinical: heart failure symptoms according to NYHA classification and/or physical examination, comorbidities (coronary artery disease, arterial hypertension, diabetes mellitus); echocardiographic: LVEDd, RVEDd, LA, RA, E/A, LVEF, valvular disorders; and technical: number of leads, number of leads passing through the tricuspid annulus, site of right ventricle stimulation (right ventricle apex - RVA; right ventricle outflow tract - RVOT; cardiac resynchronization therapy - CRT), presence of an ICD lead, time since implantation.

In both groups, the radiological picture of the lead passage through the tricuspid valve was analyzed.

\section{Statistical analysis}

In order to perform all the calculations, StatSoft Inc. (2014) Statistica (data analysis software system) 
version 12 was used. For the continuous parameters, the mean value and standard deviation were calculated. For the qualitative parameters, the absolute and percentage number of cases in relation to the whole group was presented. For the continuous values, the Shapiro-Wilk W test was used to verify whether the parameter has a normal distribution. To compare the two samples of quantitative parameters, Student's $t$-test for independent samples was performed. In the case of a non-normal distribution, the non-parametric Mann-Whitney $U$ test was performed. The $\chi^{2}$ test was used in order to compare the qualitative parameters; in case of a low number, Fisher's exact test was used. Univariate and multivariate analysis of factors influencing severe tricuspid regurgitation was performed. Using logistic regression, multivariate analysis was performed for those data which reached $p<0.1$ in univariate analysis. Multivariate analysis was performed by the backward stepwise selection method; $p<0.05$ was defined as statistically significant.

\section{Results}

The study included 100 consecutive patients (34 females, 66 males), mean age of $67.1 \pm 14$ years. Sixty-six patients had a pacemaker (PM) implanted, 27 had an implantable cardioverter-defibrillator (ICD), 1 had a pacemaker with resynchronization therapy (CRT-P), and 6 had a cardioverter-defibrillator with resynchronization therapy (CRT-D). The mean time from implantation was 9 years (1-30 years).

In the whole population, severe tricuspid regurgitation was diagnosed in 28 (28\%) patients; mean age: 71.0 \pm 16.0 years; 9 women - group A. Group B comprised 72 patients aged $65.6 \pm 13.1$ years, 25 women without severe tricuspid regurgitation (Table I).

Time from implantation did not differ between groups. The total number of leads per patient was higher in group $B$, although the number of leads crossing through the tricuspid valve was comparable between groups. The higher total number of leads in group B may be the result of the larger number of double-chamber stimulators and CRT systems in this group. In both groups, the number of ICD leads was comparable $(28.9 \%$ in group A vs. $34.7 \%$ in group B).

Patients in group A had larger diameters of the right ventricle and both atria. The groups did not differ significantly for other analyzed criteria (Table I).

Radiological signs of non-optimal lead position were visualized in 20 (71.4\%) patients in group A, and 12 (16.7\%) patients in group $B$.

As shown in Table I, radiological signs of non-optimal lead position were significantly more frequent in group A compared to group B $(p<0.0001)$. In patients without regurgitation, systems assuring atrioventricular and intraventricular synchronization (DDD and CRT) were used significantly more often.
Multivariate analysis showed that factors influencing severe tricuspid regurgitation were as follows: insufficient lead length $(O R=3.914 ; p=0.003)$ or excessive lead length $(O R=2.595 ; p=0.017)$ in the radiological picture; enlarged right ventricle in four-chamber view $(O R=1.205 ; p=0.047)$; and enlarged right atrium $(O R=$ $1.175 ; p=0.001)$.

\section{Discussion}

Tricuspid valve regurgitation is an insidious disease because most often it is (as in most of our population) asymptomatic [8, 9].

The mechanisms of tricuspid regurgitation after implantation of endocardial leads have not yet been fully discovered. Presently, prospective studies are being carried out in order to answer the question how endocardial leads influence the tricuspid valve [10]. It was hypothesized that systolic dyssynchrony resulting in negative ventricle remodeling and tricuspid annulus dilation may explain the more frequent occurrence of regurgitation in the case of apical stimulation or impaired diastolic left ventricle function [11, 12]. In our study, neither stimulation site nor left ventricle systolic function significantly influenced tricuspid regurgitation.

Other theories have suggested that tricuspid regurgitation may be caused by the mechanical influence of the lead on the tricuspid apparatus [11, 13]. In this case, a higher rate of lesions would be expected in patients with ICD leads or a large number of leads. In our material, as in the study by Al-Bawardy et al., this relation was not proved [4].

Tricuspid valve function also depends on the lead curves as it passes through the valve. In the available literature, we did not find a study correlating the radiological and echocardiographic picture after implantation of the stimulation system. We used novel definitions of excessive and insufficient lead length in the radiological chest picture, which was altogether named a "non-optimal radiological lead position".

In our population, insufficient lead length was associated with higher risk of tricuspid regurgitation than its excessive length. It seems that a stretched lead "holding" the tricuspid leaflet has higher risk of causing regurgitation than excessive lead loops inside the valve.

Patients who were admitted to the study were, by definition, asymptomatic, presenting for a routine follow-up at different times since implantation. Therefore, it is impossible to determine whether the observed radiological changes were present since implantation or developed over time. And it is not only a limitation of the study, but also reflects a true follow-up condition, where there are no indications to perform chest X-ray in asymptomatic patients.

Patients with known pre-procedural tricuspid regurgitation were not admitted to the study. However, lack 
Table I. Comparison of demographic, clinical, radiological, and echocardiographic parameters

\begin{tabular}{|c|c|c|c|c|}
\hline Parameter & Group A & Group B & $P$-value & Total \\
\hline Number of patients & 28 & 72 & - & 100 \\
\hline Age, mean \pm SD [years] & $71.0 \pm 16.0$ & $65.6 \pm 13.1$ & 0.0193 & $67.1 \pm 14.1$ \\
\hline Time since implantation, mean \pm SD [years] & $9.96 \pm 6.20$ & $8.62 \pm 5.92$ & 0.2450 & $9.00 \pm 6.00$ \\
\hline Total number of leads per patient, mean \pm SD & $1.54 \pm 0.58$ & $1.90 \pm 0.61$ & 0.0072 & $1.80 \pm 0.62$ \\
\hline $\mathrm{DDD} / \mathrm{CRT}$ & $15 / 28$ & $56 / 72$ & 0.0166 & $71 / 100(71.0 \%)$ \\
\hline ICD & $7 / 28$ & $25 / 72$ & 0.3494 & $32 / 100(32.0 \%)$ \\
\hline RVA stimulation (\%) & $19 / 28(67.9 \%)$ & $38 / 72(52.8 \%)$ & 0.2532 & $57 / 100(57.0 \%)$ \\
\hline RVOT and CRT stimulation (\%) & $9 / 28(32.1 \%)$ & $34 / 72(47.2 \%)$ & 0.2532 & $43 / 100(43.0 \%)$ \\
\hline Excessive lead length in X-ray & $8 / 28(28.6 \%)$ & $8 / 72(11.1 \%)$ & 0.0325 & $16 / 100(16.0 \%)$ \\
\hline Insufficient lead length in X-ray & $12 / 28(42.8 \%)$ & $4 / 72(5.6 \%)$ & $<0.0001$ & $16 / 100(16.0 \%)$ \\
\hline Excessive or insufficient lead length in X-ray & $20 / 28(71.4 \%)$ & $12 / 72(16.7 \%)$ & $<0.0001$ & $32 / 100(32.0 \%)$ \\
\hline Heart failure symptoms (\%) & $6 / 28(21.4 \%)$ & 8/72 (11.1\%) & 0.3105 & $14 / 100(14.0 \%)$ \\
\hline LVEF, mean \pm SD (\%) & $46.3 \pm 18.4$ & $48.9 \pm 18.2$ & 0.4055 & $48.2 \pm 18.2$ \\
\hline LVEDd, mean \pm SD [mm] & $54.2 \pm 7.3$ & $55.8 \pm 9.2$ & 0.5668 & $55.3 \pm 8.7$ \\
\hline RVEDd1, mean \pm SD $[\mathrm{mm}]$ & $33.6 \pm 6.0$ & $28.5 \pm 4.0$ & $<0.0001$ & $30.0 \pm 5.2$ \\
\hline RVEDd2, mean \pm SD [mm] & $46.5 \pm 8.8$ & $39.0 \pm 7.7$ & 0.0001 & $41.1 \pm 8.7$ \\
\hline RVEDd3, mean \pm SD [mm] & $29.0 \pm 7.9$ & $25.5 \pm 5.5$ & 0.0288 & $26.4 \pm 6.4$ \\
\hline RVEDd4, mean \pm SD [mm] & $64.2 \pm 7.8$ & $57.1 \pm 9.1$ & 0.0001 & $59.1 \pm 9.3$ \\
\hline $\mathrm{LA}$, mean $\pm \mathrm{SD}[\mathrm{mm}]$ & $52.5 \pm 8.1$ & $45.2 \pm 5.2$ & $<0.0001$ & $47.2 \pm 7.0$ \\
\hline RA1, mean \pm SD $[\mathrm{mm}]$ & $57.7 \pm 9.4$ & $44.5 \pm 6.9$ & $<0.0001$ & $48.2 \pm 9.7$ \\
\hline $\mathrm{RA} 2$, mean $\pm \mathrm{SD}[\mathrm{mm}]$ & $57.7 \pm 10.3$ & $44.5 \pm 6.3$ & $<0.0001$ & $48.2 \pm 9.6$ \\
\hline$E / A$, mean $\pm S D$ & $1.13 \pm 0.74$ & $1.18 \pm 0.86$ & 0.6684 & $1.17 \pm 0.83$ \\
\hline Coronary artery disease (\%) & $12 / 28(42.9 \%)$ & 24/72 (33.3\%) & 0.3730 & $36 / 100(36.0 \%)$ \\
\hline Arterial hypertension (\%) & 19/28 (67.9\%) & $36 / 72(50.0 \%)$ & 0.1070 & $55 / 100(55.0 \%)$ \\
\hline Diabetes mellitus (\%) & 9/28 (32.1\%) & 8/72 (11.1\%) & 0.0119 & $17 / 100(17.0 \%)$ \\
\hline
\end{tabular}

$D D D / C R T$ - dual chamber pacing system/cardiac resynchronization therapy, ICD - implantable cardioverter-defibrillator, RVA - right ventricular apex, RVOT - right ventricle outflow tract, LVEF - left ventricle ejection fraction, LVEDd - left ventricle end diastolic diameter, RVEDd1 - end diastolic right ventricle diameter in parasternal longitudinal view, RVEDd2 - end diastolic right ventricle diameter in four-chamber apical view at heart base, RVEDd3 - end diastolic right ventricle diameter in four-chamber apical view at chordae tendineae attachment, RVEDd4 - end diastolic right ventricle diameter in four-chamber apical view in longitudinal axis, LA - left atrium, RA1 - right atrium - longitudinal diameter, RA2 - right atrium - horizontal diameter, E/A - E/A assessment.

of a pre-procedural full echocardiographic study and repeated echocardiographic examinations (which are not required in a routine follow-up) made it impossible to assess some of the observed changes - enlargement of the right heart chambers, or slight changes in tricuspid regurgitation level - which is undoubtedly a limitation of the study.

\section{Conclusions}

Significant tricuspid regurgitation is a common finding in the presence of endocardial leads. Presence of tri- cuspid regurgitation seems to correlate with non-optimal radiological lead position.

\section{Conflict of interest}

Anna Rydlewska - educational grants from Medtronic, other authors declare co conflict of interest.

\section{References}

1. Al-Mohaissen MA, Chan KL. Tricuspid regurgitation following implantation of a pacemaker/cardioverter defibrillator. Curr Cardiol Rep 2013; 15: 357. 
2. Rogers JH, Bolling SF. The tricuspid valve: current perspective and evolving management of tricuspid regurgitation. Circulation 2009; 119: 2718-25.

3. Mediratta A, Addetia K, Yamat M, et al. 3D echocardiographic location of implantable device leads and mechanism of associated tricuspid regurgitation. JACC Cardiovasc Imaging 2014; 7: 337-47.

4. Al-Bawardy R, Krishnaswamy A, Rajeswaran J, et al. Tricuspid regurgitation and implantable devices. PACE 2015; 38: 259-66.

5. Paniagua D, Aldrich HR, Lieberman EH, et al. Increased prevalence of significant tricuspid regurgitation in patients with transvenous pacemakers leads. Am J Cardiol 1998; 82: 1130-2, A9.

6. Ellenbogen KA, Kay GN, Lau CP, Wilkoff BL. Clinical cardiac pacing, defibrillation and resynchronization therapy. Elsevier 2011: 443-516.

7. Guidelines on the management of valvular heart disease (version 2012) The Joint Task Force on the Management of Valvular Heart Disease of the European Society of Cardiology (ESC) and the European Association for Cardio-Thoracic Surgery (EACTS). Eur Heart J 2012; 33: 2451-96.

8. Al-Bawardy R, Krishnaswamy A, Bhargava M, et al. Tricuspid regurgitation in patients with pacemakers and implantable cardiac defibrillators: a comprehensive review. Clin Cardiol 2013; 36: 249-54.

9. Alizadeh A, Sanati HR, Haji-Karimi M, et al. Induction and aggravation of atrioventricular valve regurgitation in the course of chronic right ventricular apical pacing. Europace 2011; 13: 1587-90.

10. Dokainish H, Elbarasi E, Masiero S, et al. Prospective study of tricuspid valve regurgitation associated with permanent leads in patients undergoing cardiac rhythm device implantation: background, rationale and design. Glob Cardiol Sci Pract 2015; 2015: 41.

11. Fazio S, Carlomagno G. The importance of tricuspid regurgitation and right ventricular overload in ICD/CRT recipients: beside the left, beyond the left. Pacing Clin Electrophysiol 2011; 34: 1181-4.

12. Klutstein M, Balkin J, Butnaru A, et al. Tricuspid incompetence following permanent pacemaker implantation. Pacing Clin Electrophysiol 2009; 32 (Suppl. 1): S135-7.

13. Lin G, Nishimura RA, Connolly HM, et al. Severe symptomatic tricuspid valve regurgitation due to permanent pacemaker or implantable cardioverter-defibrillator leads. J Am Coll Cardiol 2005; 45: 1672-5. 